\title{
LIPUS Setting and Combined Effect With Therapeutic Exercise for Knee Osteoarthritis: A Systematic Review
}

\section{Masashi Kitano}

Morinomiya University of Medical Sciences: Morinomiya Iryo Daigaku https://orcid.org/0000-00022170-9508

\section{Yuse Okawa}

Morinomiya University of Medical Sciences Acupuncture Infomation Center

\section{Hirohisa Kawahata}

Department of Medical Technology, Morinomiya University of Medical Sciences

Shintarou Kudo ( $\nabla$ shintarou.iimt@gmail.com )

Department of Physical Therapy, Morinomiya University of Medical Sciences, Japan https://orcid.org/0000-0001-9483-1830

\section{Research}

Keywords: Knee osteoarthritis (OA), irradiation intensity, LIPUS, RCTs

Posted Date: December 10th, 2020

DOl: https://doi.org/10.21203/rs.3.rs-121886/v1

License: (1) This work is licensed under a Creative Commons Attribution 4.0 International License. Read Full License 


\section{Abstract}

[PURPOSE] Knee osteoarthritis (OA) is a chronic progressive disorder characterized by pain and decreased joint function. In the conservative treatment for the knee OA, the physiotherapy is known to be a common intervention. Recently, low-intensity pulsed ultrasound therapy (LIPUS) which has an effect on knee pain of knee OA has been used with therapeutic exercise. However, the combined effect of LIPUS with therapeutic exercise did not have enough evidence.

This study was conducted to assess both setting off the LIPUS and combined effect of the therapeutic exercise in the Knee OA.

[METHODS] PubMed, PEDro, CENTRAL were searched for RCTs on published before September 2019. The irradiation intensity, irradiation site, and treatment period of LIPUS were examined. Meta-analysis was performed to analyze the short and long term combined effect which was LIPUS with therapeutic exercise on the VAS score. Statistical calculations of both LIPUS with therapeutic exercise group and only therapeutic exercise group were compared from detailed data of the all eligible studies

[RESULTS] Seven RCTs were eligible. Both irradiation intensity and site were varied. Three RCTs were included in the meta-analysis, involving a total of 210 knees. There was no significant improvement in pain in the LIPUS with therapeutic exercise (LIPUS) group compared with the therapeutic exercise group in short-term (MD; $-6.86,95 \% \mathrm{Cl} ;-18.70$ to $4.99,12=74 \%$, heterogeneity: $\mathrm{P}=0.02$ ) and long-term (MD; $-16.0195 \% \mathrm{Cl} ;-32.03$ to $0.01, \mathrm{I} 2=82 \%$, heterogeneity: $\mathrm{P}=0.004)$ effects.

[CONCLUSIONS]We did not find consistent evidence of the effects of combined to LIPUS and therapeutic exercise for Knee OA. Because, selected trials were varied in intensity and irradiation site and treatment durations, which might contribute to the existence of heterogeneity. Furthermore, there were few RCTs that resulted in knee joint function.

The LIPUS combined therapeutic exercise found no significant decrease the knee pain than therapeutic exercise. Therefore, RCTs were recommended by well-designed intensity and irradiation site.

\section{Background}

Low intensity pulsed ultrasound therapy (LIPUS) is a type of ultrasound therapy that uses a low intensity pulsed wave to stimulate for the fracture to promote bone union. There have been positive reports on the effect of LIPUS on bone union[1-3], but there have also been negative reports in the recent systematic review that it has no effect on bone healing[4,5]. Therefore, the effect of LIPUS on fracture treatment will need to be discussed. Other effects of LIPUS, such as reduced inflammation[6], improved soft tissue microcirculation[7], and increased expression of vascular endothelial growth factor (VEGF) [8] have also been reported. Furthermore, several studies have reported that efficacy of LIPUS on knee osteoarthritis (knee OA)[9]. 
Knee $\mathrm{OA}$ is the most common articular disorder characterized by pain and decreased function. Primary knee OA contracted by chronic mechanical stimulation in the aging begins from $50 \sim 60$ years old. In the United States, approximately 14 million people have been reported to have symptomatic knee OA[10]. In addition, because knee $\mathrm{OA}$ is a chronic progressive disorder, patients with severe symptoms often undergo total knee arthroplasty (TKA). However, about $20 \%$ of patients were not satisfied with TKA [11]. Also, compared with conservative treatment, serious adverse events have been reported with TKA [12], and TKA does not always yield the expected results. In addition, about $20 \%$ of the knee OA undergo to the TKA [13]. Therefore, effective conservative treatment is expected to prevent of knee OA.

Conservative treatment of knee OA often includes physical therapy, orthosis, and therapeutic exercise, which is recommended in guidelines $[14,15]$. Ultrasound therapy, a type of physical therapy, is also often used in clinical practice because it improves knee OA pain [16] and increases range of motion[17] by using thermal and non-thermal mechanisms (mechanical effects). Recent systematic reviews and metaanalyses suggest that LIPUS is also effective in improving knee function[9] and that pulsed wave ultrasound is superior to continuous wave of it in improving function[18]. However, although physical therapy and therapeutic exercise are often used in combination in clinical setting, the efficacy of LIPUS treatment in combination with therapeutic exercise for knee OA has not been verified and remains controversial. In addition, the irradiation intensity, irradiation site, and type of outcome measures for LIPUS therapy have not been sufficiently examined in the literature.

Therefore, the aim of this study was to conduct a systematic review and meta-analysis of combination effects of LIPUS with therapeutic exercise, and to investigate for the parameters of LIPUS such as irradiation intensity, site, and type of outcome measures.

\section{Methods}

The selection of evidence, risk of bias assessment, and meta-analysis were presented according to the Preferred Reporting Items for Systematic Reviews and Meta-analyses (PRISMA) [19].

All available years of data in PubMed, Physiotherapy Evidence Database (PEDro), and Cochrane Central Register of Controlled Trials (CENTRAL) were searched for potential references. The search strategy used free text word retrieval and subject headings adapted for each database as well as relevant key words such as "ultrasound therapy" and "knee osteoarthritis" with the filter for article type set on "randomized controlled trial (RCT)." The search was conducted until September 2019.

All abstracts identified by electronic database searching were screened by two reviewers to exclude ineligible references. The remaining full articles were then retrieved and screened again for relevance.

The inclusion criteria are as follows:

(1) Randomized controlled trials

(2) Patients with knee osteoarthritis 
(3) Studies containing at least one group using LIPUS as an intervention

(4) English literature.

The exclusion criteria are as follows:

(1) Animal studies

(2) Abstract, letter, review, systematic review/meta-analysis, or case report

(3) Non-English literature.

The selected literature was reviewed and evaluated critically by two independent reviewers. The methodological quality assessment was conducted applying the Cochrane Collaboration's tool for assessing risk of bias[20].

Visual analogue scale (VAS) scores of pain on short and long term were used as outcomes in the metaanalysis. Reviewers extracted all relevant data separately. The extracted and pooled data included first author, year of publication, number of patients, LIPUS intervention duration, observation period, radiation intensity, radiation site, VAS scores, and type of therapeutic exercise.

Meta-analysis was performed using the software package RevMan 5.3, for comparison between LIPUS combined with therapeutic exercise and therapeutic exercise only. Statistical calculations were performed for all studies of interest using detailed LIPUS combined with therapeutic exercise and LIPUS and therapeutic exercise data. The LIPUS group (LIPUS and therapeutic exercise) and the therapeutic exercise group (Therapeutic exercise only) were compared using a random effects model ${ }^{21}$ ), significance was considered at $\mathrm{P}<0.05$. The reliability of the statistic is $95 \%$ (confidence interval; $\mathrm{Cl}$ ) was calculated.

Statistical heterogeneity was assessed using $P$ statistic[22]. Using a random effects model[11],significance was considered at $\mathrm{P}<0.05$.

\section{RESULTS}

In total, 173 potential records were identified through database searches, and 125 abstracts remained after deletion of duplicate records. The abstracts were then reviewed and preliminary assessed, followed by an assessment of the eligibility of 15 full-text articles[23-37]. Of the 15 cases, seven[31-37] met the selective criteria (Figure 1) and the other eight[23-30] were excluded. These eight articles were not eligible for intervention. In addition, three $[31,34,35]$ of the seven articles were used for meta-analysis.

The basic characteristics of included studies are shown in Table 1. The irradiation intensity was $40 \sim 500$ $\mathrm{mW} / \mathrm{cm}^{2}$, and there was a variation among studies. There was also variation among studies in the area of irradiation, including the medial and lateral cleft MCL, popliteal region, and pain site. Few reports of knee joint function such as muscle strength and range of motion were observed. Figures 2 and 3 show the "risk of bias" assessment for individual studies. Selective bias is unclear because all studies failed to 
confirm pre-registered outcomes. In one study, the risk of bias increased because patients were not blinded.

We evaluated the efficacy of LIPUS combined with therapeutic exercise for pain compared with therapeutic exercise only, by synthesis of three trials. Pooled VAS score did not demonstrate a short-term effect (MD; $-6.86,95 \% \mathrm{Cl} ;-18.70$ to 4.99 points, $12=74 \%$, heterogeneity: $P=0.02$ ) and a long-term effect (MD; $-16.01,95 \%$ Cl; -32.03 to 0.01 points, $I 2=82 \%$, heterogeneity: $P=0.004$ ) for pain relief with LIPUS combined with therapeutic exercise (Fig 3.4).

\section{Discussion}

This systematic review focuses on the efficacy of LIPUS in patients with knee OA. As a result, there were seven studies related to knee OA and LIPUS, and there was the dispersion between studies on the irradiation intensity, irradiation site, and treatment period of LIPUS. Three RCTs combined LIPUS and therapeutic exercise. We conducted a meta-analysis to combined effect of LIPUS and therapeutic exercise. As the result, the desensitization effect by the combined use of LIPUS and therapeutic exercise was not recognized.

In a previous meta-analysis, the analgesic effect of LIPUS on knee OA was observed[18]. However, previous studies have included RCTs combining LIPUS with therapeutic exercise. Therapeutic exercise for knee OA is effective in relieving pain in systematic review[38]. Therefore, in previous studies, the effect of LIPUS may have been masked by the effect of the pain relief of therapeutic exercise, and it has not been sufficiently examined whether the effect of LIPUS is more effective than therapeutic exercise alone. As a result of verifying the combined effect of LUPUS and therapeutic exercise, the effect of LIPUS was not recognized, and the analgesic effect of LIPUS could not be clarified. The possible causes were that therapeutic exercise was more effective than LIPUS and that there were problems with the protocol and outcome of LIPUS. Others included anterior, medial, and lateral parts of the knee, medial collateral ligament, pes anserinus, popliteal, lateral joint space, painful areas, and unknown. Therefore, there are varied the irradiation site among the studies. In some studies reporting negative effects of LIPUS, the site of irradiation was not uniform.

Irradiation intensity ranged from 40 to $500 \mathrm{~mW} / \mathrm{cm}^{2}$, with variations among studies[30-36]. There are a large number of RCTs with no evidence of radiation intensity. However, only Jia et al. adjusted the irradiation intensity at $120 \mathrm{~mW} / \mathrm{cm}^{2}$ referring to a previous study[39] in which the effect of LIPUS was clarified from animal experiments. Previous studies in this animal experiments have reported a reduction in inflammatory mediators and in joint effusion. Therefore, a setting of the irradiation intensity of 120 $\mathrm{mW} / \mathrm{cm} 2$ may be effective.

There are four studies which set two week treatment durations, and three studies which set eight week treatment durations. 
There are clarified only short term effects of treatment, because knee OA is chronic and progressive musculoskeletal disorder. And animal experiments have shown that LIPUS significantly suppresses inflammatory mediators and joint edema even after 8 weeks of treatment. Consideration should be given to setting the duration of treatment, as the length of treatment may affect the response to treatment.

Many of the outcomes of the selected RCTs confirmed reports of pain, Western Ontario and McMaster Universities Osteoarthritis Index (WOMAC), and gait. Knee OA has been reported to decrease gait function with pain and the knee function[40]. However, there are few RCTs that outcome the knee functions such as muscle strength and range of motion, and the effect of LIPUS on the knee function has not been fully understood. Therefore, further reports are needed to RCTs were recommended with well-designed intensity and irradiation site.

There is no clear evidence regarding the number of LIPUS interventions and the duration of LIPUS intervention. The analgesic effect of LIPUS could not be confirmed. However, there is a variation in irradiation intensity, irradiation site, and period between studies, so that the possibility of a difference in pain relief effect cannot be denied. Second, differences in the intensity, location, and duration of radiation between studies may have resulted in differences in pain relief. It is necessary to verify the combined effect of therapeutic exercise with outcome other than intensity, site, duration and pain.

There are several limitations in this study.

First, the intensity and type of therapeutic exercise in the three RCTs selected for the meta-analysis were different and high methodological heterogeneity. Second, the number of RCTs is small and publication bias cannot be assessed.

\section{Conclusion}

The desensitization effect by the combined use of LIPUS and therapeutic exercise could not be recognized. This was thought to be due to variations in the irradiation site and intensity settings. In addition, there are few studies in which changes in the structure of the knee joint are considered as an outcome, and further studies are expected.

\section{List Of Abbreviations}

CENTRAL Cochrane Central Register of Controlled Trials

Knee OA Knee osteoarthritis

LIPUS Low intensity pulsed ultrasound

PEDro Physiotherapy Evidence Database

PRISMA The Preferred Reporting Items for Systematic Reviews and Meta-analyses 
RCTs Randomized clinical trials

TE Therapeutic exercise

TKA Total knee arthroplasty

US Ultrasound

VAS Visual analog scale

VEGF Vascular endothelial growth factor

WOMAC Western Ontario and McMaster Universities Osteoarthritis Index

\section{Declarations}

\section{Ethics approval and consent to participate}

Not applicable.

\section{Consent for publication}

Not applicable.

\section{Availability of data and materials}

All data generated or analysed during this study are included in this published article and its supplementary information files.

\section{Competing interests}

The authors declare that they have no competing interests.

\section{Funding}

No external funding was received.

\section{Authors' contributions}

Conceived ideas or experimental design: MK, SK.

Searching: MK and SK.

Data analysis and interpretation: MK, YO, and SK.

Wrote the manuscript:MK. 
Supervised the study and edited the manuscript:YO,HK,and SK.

\section{Acknowledgements}

We gratefully acknowledge the work of past and present members of our laboratory.

\section{References}

1. Rutten S, Van Den Bekerom MPJ, Sierevelt IN, Nolte PA. Enhancement of bone-healing by lowintensity pulsed ultrasound: A systematic review. JBJS Rev. 2016;4(3):1-11.

2. Raza H, Saltaji H, Kaur H, Flores-Mir C, El-Bialy T. Effect of low-intensity pulsed ultrasound on distraction osteogenesis treatment time: A meta-analysis of randomized clinical trials. J Ultrasound Med. 2016;35(2):349-358.

3. Leighton R, Watson JT, Giannoudis P, Papakostidis C, Harrison A, Steen RG. Healing of fracture nonunions treated with low-intensity pulsed ultrasound (LIPUS): A systematic review and metaanalysis. Injury. 2017;48(7):1339-1347.

4. Schandelmaier S, Kaushal A, Lytvyn L, et al. Low intensity pulsed ultrasound for bone healing: systematic review of randomized controlled trials. BMJ. 2017;356(March 2018):j656.

5. Lou S, Lv H, Li Z, Tang P, Wang Y. Effect of low-intensity pulsed ultrasound on distraction osteogenesis: A systematic review and meta-analysis of randomized controlled trials. J Orthop Surg Res. 2018;13(1):1-10.

6. Nakamura T, Fujihara S, Yamamoto-Nagata K, Katsura T, Inubushi T, Tanaka E. Low-intensity pulsed ultrasound reduces the inflammatory activity of synovitis. Ann Biomed Eng. 2011;39(12):2964-2971.

7. Carow JB, Carow J, Gueorguiev B, et al. Soft tissue micro-circulation in the healthy hindfoot: a crosssectional study with focus on lateral surgical approaches to the calcaneus. Int Orthop. 2018;42(11):2705-2713.

8. Hanawa K, Ito K, Aizawa K, et al. Low-intensity pulsed ultrasound induces angiogenesis and ameliorates left ventricular dysfunction in a porcine model of chronic myocardial ischemia. PLoS One. 2014;9(8):1-11.

9. Zhou XY, Zhang XX, Yu GY, et al. Effects of low-intensity pulsed ultrasound on knee osteoarthritis: A meta-analysis of randomized clinical trials. Biomed Res Int. 2018;2018.

10. Deshpande BR, Katz JN, Solomon DH, et al. The Number of Persons With Symptomatic Knee OA_Arthritis Care and Research. Arthritis Care Res.

11. Bourne RB, Chesworth BM, Davis AM, Mahomed NN, Charron KDJ. Patient satisfaction after total knee arthroplasty: Who is satisfied and who is not? Clin Orthop Relat Res. 2010;468(1):57-63.

12. Skou ST, Roos EM, Laursen MB, et al. A randomized, controlled trial of total knee replacement. N Engl J Med. 2015;373(17):1597-1606.

13. Su X, Li C, Liao W, et al. Comparison of Arthroscopic and Conservative Treatments for Knee Osteoarthritis: A 5-Year Retrospective Comparative Study. Arthrosc - J Arthrosc Relat Surg. 
2018;34(3):652-659.

14. Zhang W, Moskowitz RW, Nuki G, et al. OARSI recommendations for the management of hip and knee osteoarthritis, Part II: OARSI evidence-based, expert consensus guidelines. Osteoarthr Cartil. 2008;16(2):137-162.

15. McAlindon TE, Bannuru RR, Sullivan MC, et al. OARSI guidelines for the non-surgical management of knee osteoarthritis. Osteoarthr Cartil. 2014;22(3):363-388.

16. Ter Haar G. Therapeutic ultrasound. Eur J Ultrasound. 1999;9(1):3-9.

17. Van Der Windt DAWM, Van Der Heijden GJMG, Van Den Berg SGM, Ter Riet G, De Winter AF, Bouter LM. Ultrasound therapy for musculoskeletal disorders: A systematic review. Pain. 1999;81(3):257271.

18. Zeng C, Li H, Yang T, et al. Effectiveness of continuous and pulsed ultrasound for the management of knee osteoarthritis: A systematic review and network meta-analysis. Osteoarthr Cartil. 2014;22(8):1090-1099.

19. Moher D, Liberati A, Tetzlaff J, Altman DG, Group TP. Preferred Reporting Items for Systematic Reviews and Meta-Analyses: The PRISMA Statement. 2009;6(7).

20. Higgins JPT, Green S, editors. Cochrane Handbook for Systematic Reviews of Interventions [Internet]. Version 5.1.0 [updated March 2011]. Cochrane Collaboration; 2011. Available from: www.cochranehandbook.org

21. Higgins JPT, Thompson SG, Deeks JJ, Altman DG. Measuring inconsistency in meta-analyses. Br Med J. 2003;327(7414):557-560.

22. Deeks JJ, Higgins JP, Altman DG. Analysing data and undertaking meta-analyses. Cochrane Handb Syst Rev Interv. 2019:241-284.

23. Boyaci A, Tutoglu A, Boyaci N, Aridici R, Koca I. Comparison of the efficacy of ketoprofen phonophoresis, ultrasound, and short-wave diathermy in knee osteoarthritis. Rheumatol Int. 2013;33(11):2811-2818.

24. Cetin N, Aytar A, Atalay A, Akman MN. Comparing hot pack, short-wave diathermy, ultrasound, and TENS on isokinetic strength, pain, and functional status of women with osteoarthritic knees: A singleblind, randomized, controlled trial. Am J Phys Med Rehabil. 2008;87(6):443-451.

25. Draper DO, Klyve D, Ortiz R, Best TM. Effect of low-intensity long-duration ultrasound on the symptomatic relief of knee osteoarthritis: A randomized, placebo-controlled double-blind study. $\mathrm{J}$ Orthop Surg Res. 2018;13(1):1-9.

26. Falconer J, Hayes KW, Chang RW. Effect of ultrasound on mobility in osteoarthritis of the knee.A randomized clinical trial. Arthritis Rheum. 1992;5(1):29-35.

27. Kozanoglu E, Basaran S, Guzel R, Guler-Uysal F. Short term efficacy of ibuprofen phonophoresis versus continuous ultrasound therapy in knee osteoarthritis. Swiss Med Wkly. 2003;133(23-24):333338. 
28. Luksurapan W, Boonhong J. Effects of phonophoresis of piroxicam and ultrasound on symptomatic knee osteoarthritis. Arch Phys Med Rehabil. 2013;94(2):250-255.

29. Mascarin NC, Vancini RL, Andrade MDS, Magalhães EDP, De Lira CAB, Coimbra IB. Effects of kinesiotherapy, ultrasound and electrotherapy in management of bilateral knee osteoarthritis: Prospective clinical trial. BMC Musculoskelet Disord. 2012;13.

30. Yang P fei, Li D, Zhang S mo, et al. Efficacy of ultrasound in the treatment of osteoarthritis of the knee. Orthop Surg. 2011;3(3):181-187.

31. Huang MH, Lin YS, Lee CL, Yang RC. Use of ultrasound to increase effectiveness of isokinetic exercise for knee osteoarthritis. Arch Phys Med Rehabil. 2005;86(8):1545-1551.

32. Tascioglu F, Kuzgun S, Armagan O, Ogutler G. Short-term effectiveness of ultrasound therapy in knee osteoarthritis. J Int Med Res. 2010;38(4):1233-1242.

33. Loyola-Sánchez A, Richardson J, Beattie KA, Otero-Fuentes C, Adachi JD, Maclntyre NJ. Effect of lowintensity pulsed ultrasound on the cartilage repair in people with mild to moderate knee osteoarthritis: A double-blinded, randomized, placebo-controlled pilot study. Arch Phys Med Rehabil. 2012;93(1):35-42. doi:10.1016/j.apmr.2011.07.196

34. Cakir S, Hepguler S, Ozturk C, Korkmaz M, Isleten B, Atamaz FC. Efficacy of therapeutic ultrasound for the management of knee osteoarthritis: A randomized, controlled, and double-blind study. Am J Phys Med Rehabil. 2014;93(5):405-412.

35. Kapci Yildiz S, Ünlü Özkan F, Aktaş I, Şilte AD, Yilmaz Kaysin M, Bilgin Badur N. The effectiveness of ultrasound treatment for the management of knee osteoarthritis: A randomized, placebo-controlled, double-blind study. Turkish J Med Sci. 2015;45(6):1187-1191.

36. Jia L, Wang Y, Chen J, Chen W. Efficacy of focused low-intensity pulsed ultrasound therapy for the management of knee osteoarthritis: A randomized, double blind, placebo-controlled trial. Sci Rep. 2016;6(October):1-9.

37. Kim ED, Won YH, Park SH, et al. Efficacy and safety of a stimulator using low-intensity pulsed ultrasound combined with transcutaneous electrical nerve stimulation in patients with painful knee osteoarthritis. Pain Res Manag. 2019;2019.

38. Imoto AM, Pardo JP, Brosseau L, et al. Evidence synthesis of types and intensity of therapeutic landbased exercises to reduce pain in individuals with knee osteoarthritis. Rheumatol Int. 2019; (0123456789).

39. Jia L, Chen J, Wang Y, Zhang Y, Chen W. Focused Low-intensity Pulsed Ultrasound Affects Extracellular Matrix Degradation via Decreasing Chondrocyte Apoptosis and Inflammatory Mediators in a Surgically Induced Osteoarthritic Rabbit Model. Ultrasound Med Biol. 2016;42(1):208-219.

40. Van Tunen JAC, Dell'Isola A, Juhl C, et al. Association of malalignment, muscular dysfunction, proprioception, laxity and abnormal joint loading with tibiofemoral knee osteoarthritis - A systematic review and meta-analysis. BMC Musculoskelet Disord. 2018;19(1).

\section{Tables}


Due to technical limitations, table 1 is only available as a download in the Supplemental Files section.

\section{Figures}

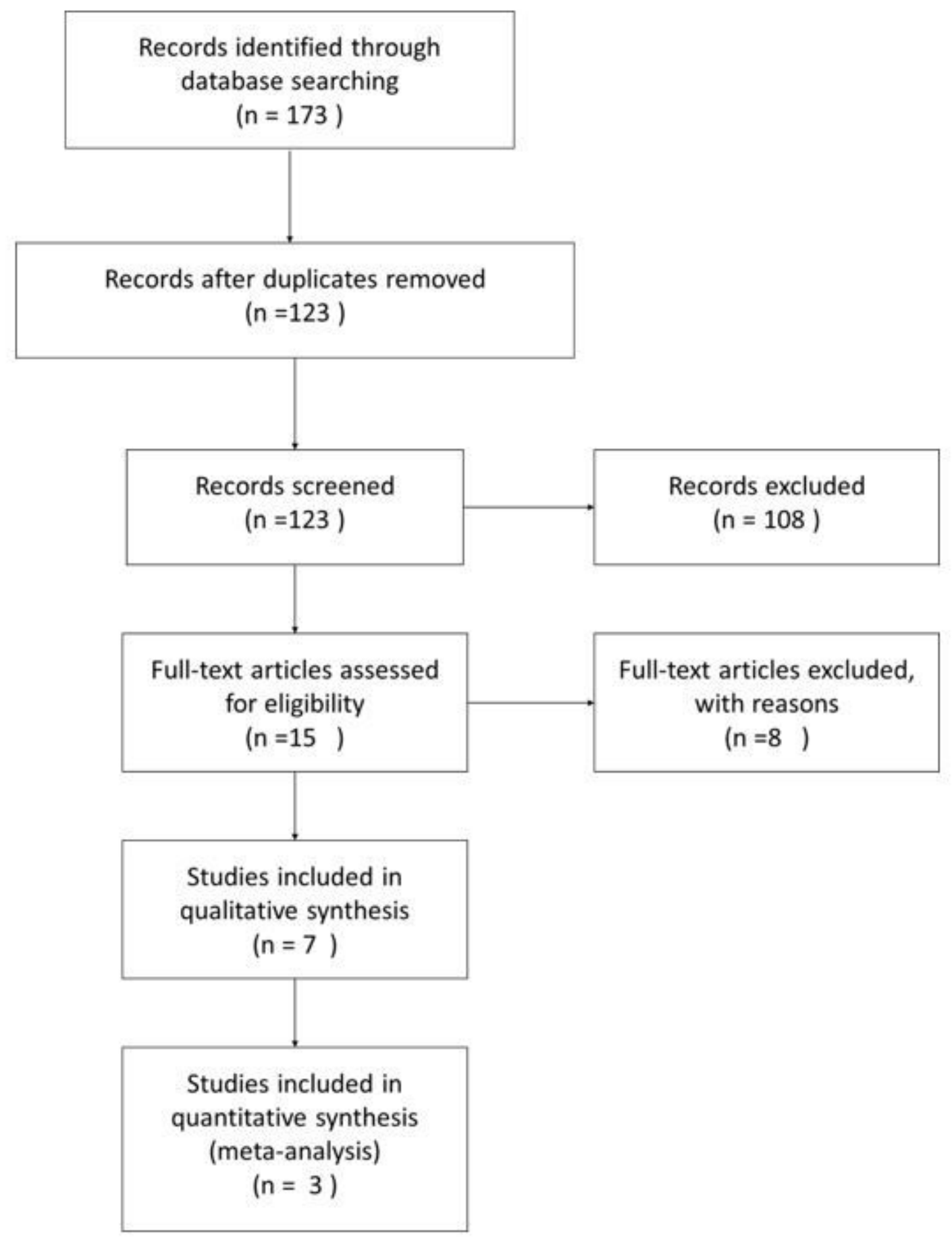

\section{Figure 1}

Flowchart for the selection of included trials. 


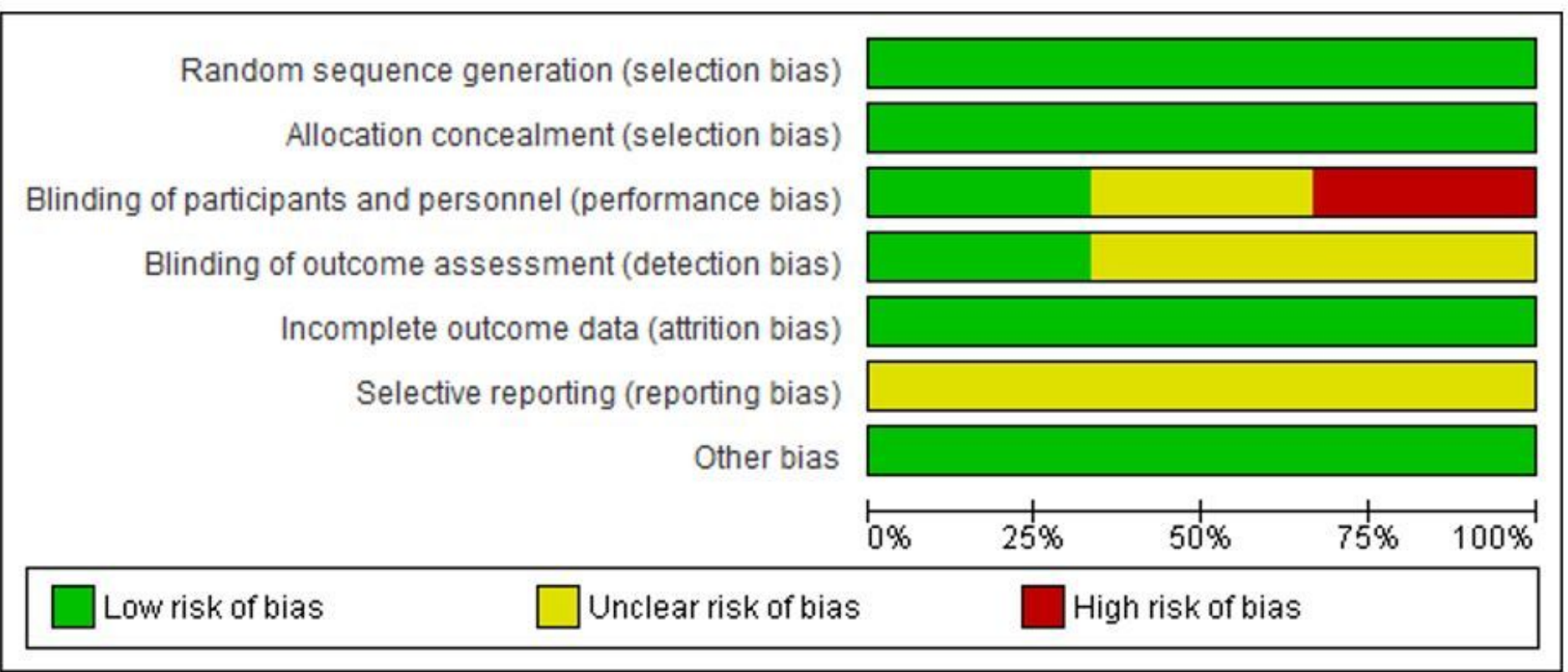

Figure 2

Risk of bias graph: review authors' judgements about each risk of bias item presented as percentages across all included studies. 


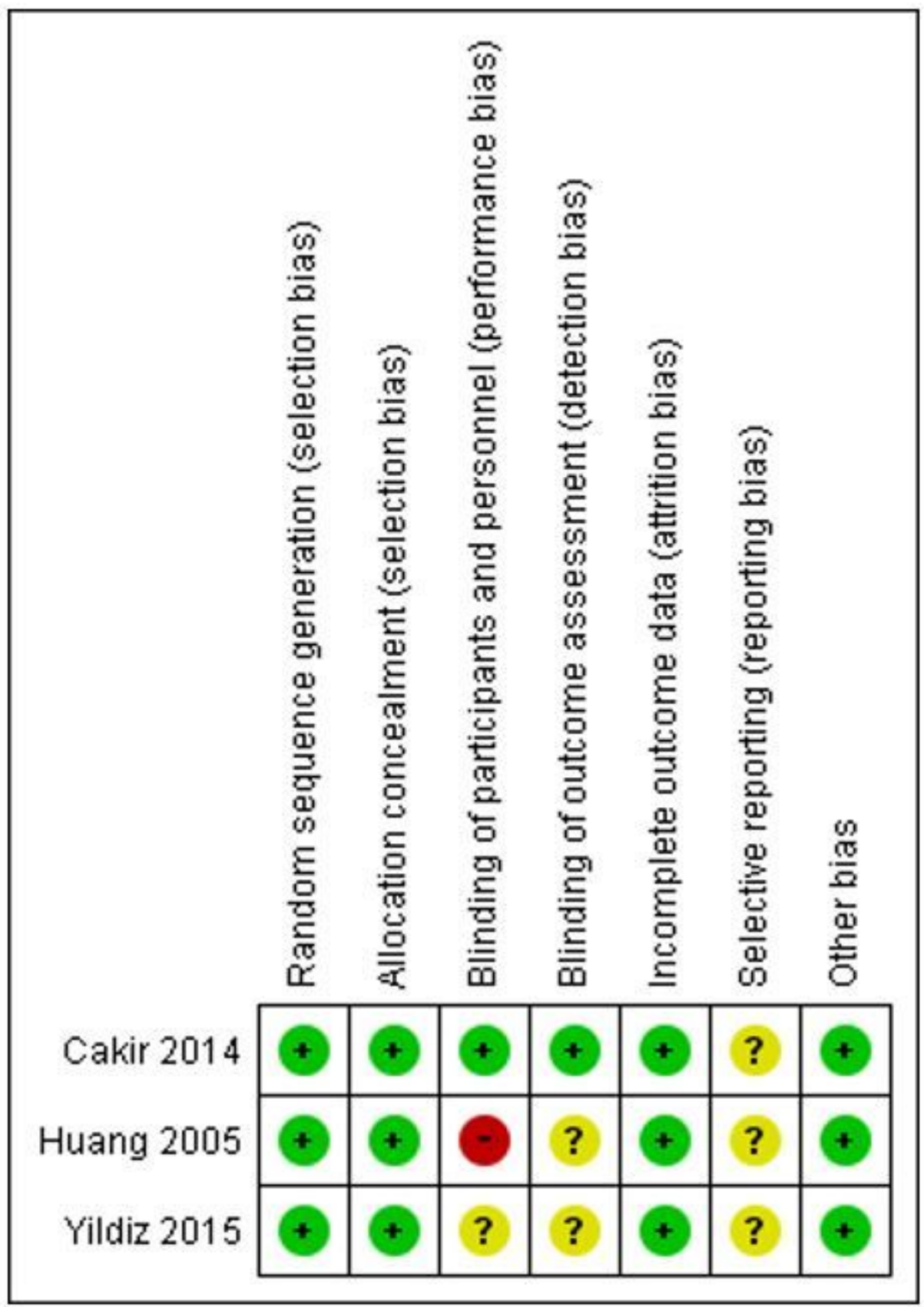

Figure 3

Risk of bias summary: review authors' judgements about each risk of bias item for each included study.

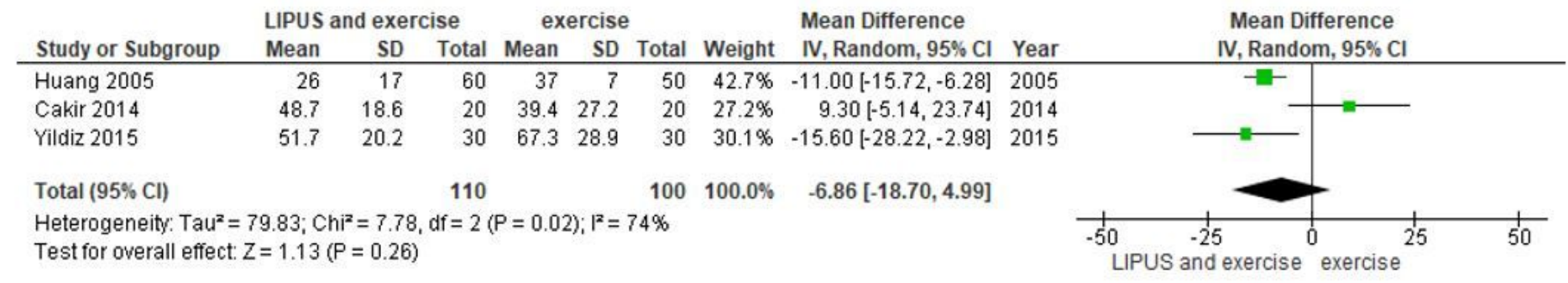




\section{Figure 4}

short-term effect

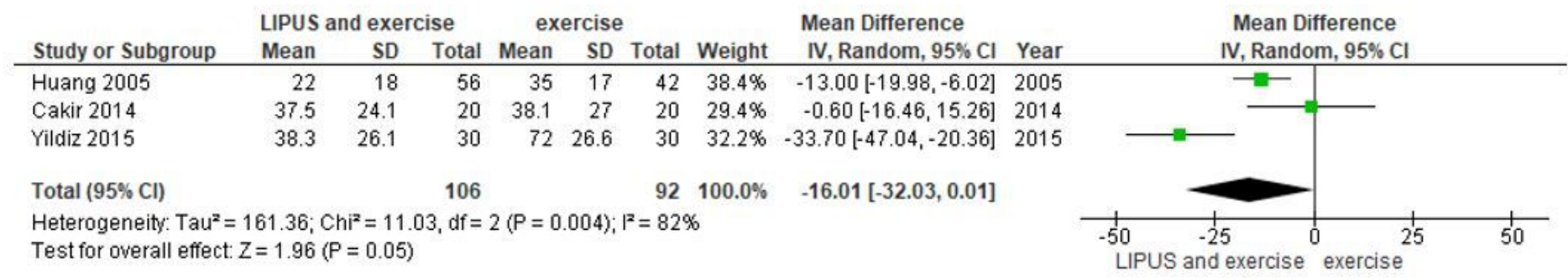

\section{Figure 5}

long-term effect

\section{Supplementary Files}

This is a list of supplementary files associated with this preprint. Click to download.

- PRISMAPchecklist.docx

- AppendixA.docx

- Table1.jpg 